\title{
EFFECT OF THE RADIATIVE DAMPING ON MAGNETOHYDRODYNAMIC WAVES IN AN ISOTHERMAL MEDIUM
}

\author{
HADI ALKAHBY, ANDREW TALMADGE, and ABRAHAM JALBOUT
}

\author{
Received 30 April 2001
}

\begin{abstract}
We investigate the effect of the heat radiation on the reflection and dissipation of upward propagating waves in an isothermal atmosphere. It is shown that the magnetic field produces a totally reflecting layer. Consequently, the atmosphere can be divided into two distinct regions. In the lower region, the solution can be written as a linear combination of an upward and a downward propagating wave, and in the upper region the solution, which satisfies the upper boundary condition, decays exponentially or behaves like a constant. These two regions are connected by a region in which the reflection and transmission of the waves takes place. Moreover, the heat radiation affects only the lower region and changes the sound speed from the adiabatic value to the isothermal one. The reflection coefficient and the attenuation factor of the amplitude of the waves are derived for all values of the heat radiation coefficient. Finally, the conclusions are presented in connection with the heating process of the solar atmosphere.
\end{abstract}

2000 Mathematics Subject Classification: 76N15.

1. Introduction. Upward propagating small amplitude magnetohydrodynamic waves in an isothermal and nonisothermal atmospheres have been extensively studied in recent years. The motivation of these studies is due mainly to the application of these waves to phenomena in compressible ionized fluids such as solar, stellar, and planetary atmospheres and to certain phenomena in ocean dynamics (see $[1,2,3,7,9,10,11,12,13]$ and the references therein). In this paper, a linearized theory of magnetoatmospheric waves, involving the combined effect of restoring forces due to compressibility, magnetic pressure, and radiative damping is developed for the case of a uniform horizontal magnetic field. A full wave equation is derived and then reduced to a special and important case of an isothermal atmosphere with a uniform horizontal magnetic field and radiative damping. It is shown that the presence of a uniform horizontal magnetic field in an isothermal atmosphere produces a reflecting and nonabsorbing critical layer. As a result, the atmosphere is divided into two distinct regions, above and below the reflecting layer. Below the reflecting layer, the solution can be written as a linear combination of an upward and a downward propagating wave with equal wavelengths and equal attenuation factor in the amplitude of the wave. Above the reflecting critical layer, the solution satisfying the prescribed boundary condition either decays exponentially with altitude or behaves like a constant. These two distinct regions are connected by a critical layer and in it the reflection, tunneling, and wave modification take place. In addition, it is shown that 
when the heat radiation is intense, the sound speed changes from the adiabatic value to the isothermal one. Consequently, the wave length is changed from the adiabatic value to the isothermal one. As a result, the propagation process below the reflective layer will be changed to an isothermal one and the trapped wave will dissipate its energy which will contribute to the heating of the atmosphere. This effect is of particular interest in solar activity in the sunspots because of their strong magnetic field. In addition, it is shown that the magnetic field lines is affected by the heat flow but not by the heat existence.

To obtain a unique solution, certain conditions must be imposed. For this reason we are able to introduce and justify the magnetic energy as an upper boundary condition. Finally, the reflection coefficient, location of the critical layer, attenuation factor of the wave amplitude, and an equation for the resonance are determined and the results are analyzed in connection with the heating process of the solar atmosphere.

2. Mathematical formulation of the problem. Suppose that an atmosphere, which is inviscid and perfectly electrically conducting, occupies the upper half-space $z>0$. It is assumed that the gas is under the influence of a horizontal magnetic field and a uniform gravitational acceleration in the negative $z$ direction. Let $p, \rho, u, T$, and $B$ be the perturbation quantities in the pressure, density, velocity, temperature, and magnetic field strength. Let $p_{0}, \rho_{0}, u_{0}, T_{0}$, and $B_{0}$ be the equilibrium quantities. The equations of motion are

$$
\begin{gathered}
\rho_{0} \frac{\partial u}{\partial t}+\nabla p-\rho g-\frac{1}{4 \pi}\left[(\nabla \times B) \times B_{0}+\left(\nabla \times B_{0}\right) \times B\right]=0 \\
\frac{\partial \rho}{\partial t}+\nabla \cdot\left(\rho_{0} u\right)=0, \quad \frac{\partial B}{\partial t}-\nabla \times\left(u \times B_{0}\right)=0 \\
\frac{\partial \rho}{\partial t}+u \cdot \nabla p_{0}-c_{o}^{2}\left(\frac{\partial \rho}{\partial t}+u \cdot \nabla \rho_{0}\right)+\frac{p_{0}}{\tau} \frac{T}{T_{0}}=0 \\
\frac{d p_{0}}{d z}+\rho_{0} g+\frac{1}{8 \pi} \frac{d B_{0}^{2}}{d z}=0
\end{gathered}
$$

These are, respectively, the momentum equation, the mass conservation equation, the induction equation, the heat flow equation, and the magnetohydrostatic equilibrium equation. Here $c_{0}=\left(\gamma p_{0} / \rho_{0}\right)^{1 / 2}$ is the adiabatic sound speed, and $\tau$ is the radiative relaxation time. The form of $\tau$ differs depending on whether continuum or line emissions dominate the oscillatory process. If the line emissions dominate the oscillatory process, the radiative relaxation time can be written as

$$
\tau=\tau_{1}=\frac{\rho_{0} c_{p}}{16 \sigma \kappa T_{0}^{3}}
$$

where $c_{p}$ is the specific heat at constant pressure, $\sigma$ is the Stefan-Boltzmann constant, and $\kappa$ is the mean absorption coefficient. On the other hand, if the continuum emissions is dominant, the form $\tau$ will be the same except $c_{p}$ is replaced by the specific heat at a constant volume $c_{V}$. Assuming the time dependence solutions, $\exp (-i \omega t)$, for the perturbation variables, $p=p(z) \exp (-i \omega t)$, and so forth then (2.3) may be 
written in the following form:

$$
i \omega p-\frac{\left[c_{0}^{2}\left(\left(a_{2}-1\right) u \cdot \nabla \rho_{0}+a_{2} \rho_{0} \nabla \cdot u\right)+u \cdot \nabla p_{0}\right]}{a_{1}}=0,
$$

where

$$
a_{1}=1+\frac{i}{\omega \tau}, \quad a_{2}=1+\frac{i}{\gamma \omega \tau} .
$$

Using the Fourier time decomposition to (2.1), with aid of (2.3) and (2.6), letting the velocity $u=\left(U, V, W_{1}\right)$ and eliminating the perturbations quantities $p, \rho$, and $B$, respectively, we obtain the following vector equation for the velocity alone:

$$
\begin{aligned}
\omega \rho_{0} u & +\nabla\left[\frac{\rho_{0}}{a_{1}} W_{1}\left(g+\frac{1}{2}\left[\frac{d a_{0}^{2}}{d z}-\frac{a_{0}^{2}}{H}\right]\right)+\frac{a_{2}}{a_{1}} c_{0}^{2} \rho_{0} \nabla \cdot u-\frac{c_{0}^{2} \rho_{0}\left(a_{2}-1\right)}{H a_{1}} W_{1}\right]-\left[u \nabla \rho_{0}+\rho_{0} \nabla \cdot u\right] g \\
& +\frac{1}{4 \pi}\left\{\left(\nabla \times B_{0}\right) \times\left(\nabla \times\left(\nabla \times B_{0}\right)\right)-B_{0} \times\left(\nabla \times\left[\nabla \times\left(u \times B_{0}\right)\right]\right)\right\}=0,
\end{aligned}
$$

where $H=-\rho_{0} /\left(d \rho_{0} / d z\right)$ is the density scale height, and $a_{0}=B_{0} /\left(4 \pi \rho_{0}\right)^{1 / 2}$ is the Alfvén speed. Removing the horizontal dependence of the velocity by assuming $u=$ $u(z) \exp \left[i\left(k_{x} x+k_{y} y\right)\right]$, the three components of (2.7) form a system of three ordinary differential equations in $U, V$, and $W_{1}(z)$ (the analysis proceeds in much the same way as in [6], so we omit the details for simplicity). The horizontal components of velocity may be eliminated in favor of $W_{1}$ and ultimately, a second-order equation in the vertical velocity alone is obtained

$$
\frac{d^{2} W_{1}}{d z^{2}}+A(z) \frac{d W_{1}}{d z}+B(z) W_{1}=0 .
$$

The coefficients $A(z)$ and $B(z)$ are defined by

$$
\begin{aligned}
& A(z)=\omega^{4} A_{1}^{2} \frac{d c^{2}}{d z} D^{-1} E^{-1}+A_{2} A_{1} \frac{d a_{0}^{2}}{d z} D^{-1}+\omega^{2} A_{1} D^{-1} \Psi-H^{-1}, \\
& B(z)=B_{1} \omega^{6}-B_{2} \omega^{4}+\left(B_{3}-B_{4}+B_{5}\right) \omega^{2}+B_{6}+B_{7}+B_{8} .
\end{aligned}
$$

Here we have

$$
\begin{aligned}
D & =\left(\omega^{2}-a_{0}^{2} k_{x}^{2}\right)\left[\omega^{2}\left(c^{2}+a_{0}^{2}\right)-c^{2} a_{0}^{2} k_{x}^{2}\right], \\
E & =\omega^{4}-C_{1} \omega^{2}+C_{2}, \quad A_{1}=\left(\omega^{2}-a_{0}^{2} k_{x}^{2}\right), \\
A_{2} & =\left(\omega^{2}-c^{2} k_{x}^{2}\right), \quad A_{3}=\left(1+\omega^{4} E^{-1}\right)-\omega^{4}, \\
B_{1} & =\left(1-k_{y}^{2}(g-\Psi) \frac{d a_{0}^{2}}{d z} E^{-1}\right), \\
B_{2} & =\left[\left(c^{2}+a_{0}^{2}\right)\left(k_{x}^{2}+k_{y}^{2}\right)+a_{0}^{2} k_{x}^{2}\right], \\
B_{3} & =a_{0}^{2} k_{x}^{2}\left(k_{x}^{2}+k_{y}^{2}\right)\left(2 c^{2}+a_{0}^{2}\right), \\
B_{4} & =(g-\Psi)\left(k_{x}^{2}+k_{y}^{2}\right)\left(g-c^{2} H^{-1}\right),
\end{aligned}
$$




$$
\begin{aligned}
& B_{5}=(g-\Psi) H^{-1} a_{0}^{2} k_{y}^{2}, \\
& B_{6}=-a_{0}^{2} k_{x}^{2}\left(k_{x}^{2}+k_{y}^{2}\right)\left[c^{2} a_{0}^{2} k_{x}^{2}-(g-\Psi)\left(g-c^{2} H^{-1}\right)\right], \\
& B_{7}=-(g-\Psi)\left(\omega^{2}-a_{0}^{2} k_{x}^{2}\right) \omega^{2}\left(k_{x}^{2}+k_{y}^{2}\right) \frac{d c^{2}}{d z} E^{-1}, \\
& B_{8}=-\Psi E H^{-1}-\omega^{2}\left(\omega^{2}-a_{0}^{2} k_{x}^{2}\right) \frac{d \Psi}{d z} D^{-1}, \\
& C_{1}=\left(k_{x}^{2}+k_{y}^{2}\right)\left(c^{2}+a_{0}^{2}\right) \\
& C_{2}=\left(k_{x}^{2}+k_{y}^{2}\right) c^{2} a_{0}^{2} k_{x}^{2} \\
& C^{2}=\frac{a_{2}}{a_{1}} c_{0}
\end{aligned}
$$

and $\Psi$ denotes the scaled temperature gradient which is defined by the equation

$$
\Psi=\left(1-\frac{1}{a_{1}}\right)\left(\frac{1}{2}\left[\frac{d a_{0}^{2}}{d z}-\frac{a_{0}^{2}}{H}\right]+g\right)+\frac{a_{2}-1}{a_{1}} \frac{c_{0}^{2}}{H}=-\frac{i}{\gamma a_{1} \omega \tau} \frac{d c_{0}^{2}}{d z} .
$$

3. Simplification and boundary conditions of the problem. For the simplification of the problem, the atmosphere is assumed to be an isothermal one (i.e., the speed of sound is constant), and permeated with a uniform horizontal magnetic field $B=$ $\left(B_{0}, 0,0\right)$. Moreover, the equilibrium pressure, density, and temperature are connected by the gas law $P_{0}(z)=R T_{0} \rho_{0}$ and the hydrostatic equation $d P_{0} / d z+g \rho_{0}=0$, where $R$ denotes the gas constant. Using the gas law and the hydrostatic equations, the pressure and density can be written in the following form:

$$
\frac{P_{0}(z)}{P_{0}(0)}=\frac{\rho_{0}(z)}{\rho_{0}(0)}=\exp \left(-\frac{z}{H}\right)
$$

where $H=R T_{0} / g$ denotes the density scale height. Moreover, let $z^{*}=z / H, W=$ $H W_{1} / c$ where in the body of the problem the star notation is eliminated for simplicity. Let $k_{y}=0, a_{0}^{2}=B^{2} /\left(4 \pi \rho_{0}(0)\right), a^{2}=a_{0}^{2} / c^{2}, q=a_{2} \gamma / a_{1}, \xi_{0}=a^{2} \omega /\left(k^{2}-\omega^{2}\right), k=H k_{x}$, and assume that

$$
W_{1}(z)=W(\xi) \exp (k z), \quad \xi=\xi_{0} \exp (-z)
$$

As a result, the differential equation (2.8) can be written as

$$
\xi(1-\xi) \frac{d^{2} W(\xi)}{d \xi^{2}}+(2 k+1-(2 k+2) \xi) \frac{d W(\xi)}{d \xi}-\left(\omega^{2}+k+\frac{(q-1)}{q^{2}} \frac{k^{2}}{\omega^{2}}\right) W(\xi)=0
$$

It is clear that the differential equation (3.3) is a special case of the hypergeometric equation

$$
\xi(1-\xi) \frac{d^{2} W(\xi)}{d \xi^{2}}+(c-(a+b+1) \xi) \frac{d W(\xi)}{d \xi}-a b W(\xi)=0
$$


with

$$
a+b=c=2 k+1, \quad a b=\omega^{2}+k+\frac{(q-1)}{q^{2}} \frac{k^{2}}{\omega^{2}} .
$$

The oscillations can be assumed to be excited by some mechanism at $z=0$ or below. The exact nature of the excitation is not important because our object is to investigate the reflection and effect of the heat radiation on the propagation of the waves at high altitudes. If the atmosphere is viscous, an appropriate condition would be the dissipation condition, which requires that the energy dissipation in an infinite column of fluid of unit cross-section to be finite. Since the dissipation condition depends on the square of the gradient of the velocity, this implies that

$$
\int_{0}^{\infty}\left|\frac{d W}{d z}\right|^{2} d z<\infty
$$

In this problem the fluid is inviscid, but the integral in (3.6), which is sometimes called the upper boundary condition, is proportional to the magnetic energy in an infinite column of fluid of unit cross-section. This condition is a reasonable one to apply so long as there is no energy radiation to infinity, which is true in our case. A boundary condition is required at $z=0$ and we set

$$
W(0)=1,
$$

by suitable normalizing of $W(z)$. This condition, sometimes, is called the lower boundary condition. We show that the upper boundary condition and the lower boundary condition are sufficient to ensure a unique solution, within a multiplicative constant.

4. Solution of the simplified problem. In this section, we investigate and analyze the solutions of the following differential equation subject to the lower and upper boundary conditions given in Section 3:

$$
\xi(1-\xi) \frac{d^{2} W(\xi)}{d \xi^{2}}+(2 k+1-(2 k+2) \xi) \frac{d W(\xi)}{d \xi}-\left(\omega^{2}+k+\frac{(q-1)}{q^{2}} \frac{k^{2}}{\omega^{2}}\right) W(\xi)=0,
$$

where

$$
a+b=c=2 k+1, \quad a b=\omega^{2}+k+\frac{q-1}{q^{2}} \frac{k^{2}}{\omega^{2}}, \quad q=\frac{\gamma(\omega \tau+i)}{(\gamma \omega \tau+i)} .
$$

It is clear that when $\tau \rightarrow 0$ we have $q \rightarrow \gamma$. Consequently, the last term in (2.2), which contains the heat perturbation, increases to infinity. In other words, when the heat is intense enough, the oscillatory process will be dominated by the heat radiation. In addition, the sound speed changes from the adiabatic value $\sqrt{\gamma g H}$ to the isothermal one $\sqrt{g H}$. Moreover, when $\tau \rightarrow 0$ we obtain $a b=\omega^{2}+k+(\gamma-1) k^{2} / \gamma \omega^{2}$ and we recover (3.7) in [6]. On the other hand, when $\tau \rightarrow \infty$, the term with heat perturbation in (2.2) will be eliminated. Consequently, the oscillatory process will be adiabatic.

It is clear that the differential equation has three regular singular points $\xi=0$, $\xi=1$, and $\xi=\infty$. The intermediate singular point $\xi=1$ indicates the existence and location of the critical layer, in which the reflection and wave transformation takes 
place. In addition $\xi \rightarrow 0$ as $z \rightarrow \infty, \xi \rightarrow 1$ as $\xi_{0} \rightarrow \exp (z)$, and finally, $\xi \rightarrow \infty$ when $\omega \rightarrow k$ for a fixed value of $z$. Two singular points of the differential equation indicate that the atmosphere can be divided into the two distinct regions above and below the reflecting layer and this idea will be clear in the analysis of the solution in Section 6 . Solving for the parameters $a$ and $b$ we obtain

$$
a=\frac{1}{2}+d+i \beta, \quad b=\frac{1}{2}-d+i \beta
$$

where $d$ and $\beta$ are the real and imaginary parts of the complex number

$$
\sqrt{\left(k^{2}+\frac{1}{4}\right)-\left(\omega^{2}+\frac{4(q-1)}{q \omega^{2}}\right)} .
$$

The differential equation (4.1) has two linearly independent solutions that can be written, for $|\xi|<1$, in the following form:

$$
W_{1}(\xi)=F(a, b, c, \xi), \quad W_{2}(\xi)=\xi^{1-c} F(a-c+1, b-c+1,2-c, \xi),
$$

and $F$ denotes the hypergeometric function, which is defined by

$$
F(a, b, c, \xi)=\frac{\Gamma(c)}{\Gamma(a) \Gamma(b)} \sum_{n=0}^{\infty} \frac{\Gamma(a+n) \Gamma(b+n)}{\Gamma(c+n)} \frac{\xi^{n}}{n !},
$$

where $\Gamma$ is the gamma function. It is clear, using (3.2), that

$$
\xi^{1-c}=\left|\xi_{0} \exp (-z)\right|^{1-c} .
$$

Since $c=2 k+1$, we have $1-c=-2 k$, and hence

$$
\xi^{1-c}=\left|\xi_{0}\right| \exp (2 k z)
$$

Consequently, $W_{2}(z) \rightarrow \infty$ as $z \rightarrow \infty$, since $k>0$. As a result, $W_{2}(\xi)$ will be eliminated using the magnetic energy condition, defined by (3.6). Finally, the solution of the differential equation (3.3) that satisfies the prescribed boundary conditions can be written as

$$
W(\xi)=C W_{1}(\xi)=C F(a, b, c, \xi),
$$

where $C$ is a multiplicative constant which can be determined using the lower boundary condition.

5. Asymptotic estimate and the magnitude of the reflection coefficient. The asymptotic behavior of $F(a, b, c, \xi)$ for $|\xi|>1$ and $|\arg (-\xi)|<\pi / 2$ can be written in the following form:

$$
\begin{aligned}
W(\xi)=C[ & \frac{\Gamma(c) \Gamma(b-a)}{b \Gamma^{2}(b)}(-\xi)^{-a} F\left(a, 1-c+a, 1-b+a, \xi^{-1}\right) \\
& \left.+\frac{\Gamma(c) \Gamma(a-b)}{a \Gamma^{2}(a)}(-\xi)^{-b} F\left(b, 1-c+b, 1-a+b, \xi^{-1}\right)\right] .
\end{aligned}
$$


Retaining the most significant terms of (5.1), we have

$$
W(\xi)=C\left[\frac{\Gamma(c) \Gamma(b-a)}{b \Gamma^{2}(b)}(-\xi)^{-a}+\frac{\Gamma(c) \Gamma(a-b)}{a \Gamma^{2}(a)}(-\xi)^{-b}\right] .
$$

Reintroducing the variable $z$, using (3.2), we have

$$
W(z) \sim C\left[P\left\{\exp \left(\left(\frac{1}{2}-d+i \beta\right) z\right)+R \exp \left(\left(\frac{1}{2}+d-i \beta\right) z\right)\right\}\right],
$$

where $R$ denotes the reflection coefficient defined by

$$
\begin{aligned}
& R=\frac{b \Gamma^{2}(b) \Gamma(a-b)}{a \Gamma^{2}(a) \Gamma(b-a)} \exp \left[-(a-b) \log \left(\xi_{0}\right)\right] \\
& R=\exp \left(i \theta-(2 d-2 i \beta) \log \left(\xi_{0}\right)\right) \\
& P=\frac{\Gamma(c) \Gamma(b-a)}{b \Gamma^{2}(b)} \\
& \theta=\arg \left(\frac{b \Gamma^{2}(b) \Gamma(a-b)}{a \Gamma^{2}(a) \Gamma(b-a)}\right) .
\end{aligned}
$$

The constant $C$ can be determined by applying the lower boundary condition (3.7). Consequently, we have

$$
C=\frac{1}{P(1+R)}
$$

6. Conclusion and general remarks. (A) It is clear, in (5.3), that the solution below the reflecting layer can be written as a linear combination of an upward and a downward propagating wave with equal wave number and equal decaying factor. The first term on the right represents an upward propagating wave, its amplitude decaying with altitude like $\exp (-d z)$, while the second term is a downward traveling wave decaying in the same rate. The decaying factor will be eliminated when $\tau \rightarrow 0$ and when $\tau \rightarrow \infty$, as we indicated in the solution of the problem. At the same time, the wave number changes from $\beta$ to $\beta_{i}$.

(B) It is clear that the magnitude of the reflection coefficient, $|R|=\exp (-2 d)$, depends on the value of the decaying factor $d$. It follows from (A) that the magnitude of the reflection coefficient is one when the radiative damping is zero and when it goes to infinity.

(C) It has been reported, see [6], that when the isothermal atmosphere is influenced by a uniform horizontal magnetic field, the magnitude of the reflection coefficient is one. This result is expected because of the dissipationless nature of the magnetic field. It flows from (A) and (B) that the magnitude of the reflection coefficient is not effected by the heat radiation when the atmosphere is adiabatic or isothermal and it is less than one only when the atmosphere is in process of change from adiabatic to isothermal form and vice versa. In other words, the magnetic field line could be disturbed by the heat flow not by the heat existence. This property should be investigated experimentally to determine the disturbance of the magnetic field lines.

(D) An equation for the resonance can be derived when the atmosphere is adiabatic and when it is isothermal. The resonance occurs if the magnetic field strength is 
matched with wave length of the wave for infinitely many values of the magnetic field and the frequency of the wave $[1,3,4,6,7,8]$ and a numerical computation can be found in $[4,5]$. At the resonance frequencies the values of the kinetic and magnetic energies of the wave will be increase to a very large values.

REMARK 6.1. Conclusion (C) is a theoretical prediction and can be a very interesting experimental work to determine the form of the disturbance of the magnetic field during the flow.

\section{REFERENCES}

[1] H. Y. Alkahby, On the coronal heating mechanism by the resonant absorption of Alfvén waves, Int. J. Math. Math. Sci. 16 (1993), no. 4, 811-816.

[2] _ Reflection and dissipation of hydromagnetic waves in a viscous and thermally conducting isothermal atmosphere, Geophys. Astrophys. Fluid Dynam. 72 (1993), 197-207.

[3] H. Y. Alkahby and T. Hussain, The heating of the solar corona by the resonant absorption of Alfvén waves, Appl. Math. Lett. 6 (1993), no. 6, 59-63.

[4] H. Y. Alkahby and F. N. Jalbout, Numerical studies of vertically propagating acoustic and magneto-acoustic waves in an isothermal atmosphere, Proceedings of the 15th Annual Conference of Applied Mathematics (Edmond, OK, 1999), Southwest Texas State University, Texas, 1999, pp. 93-103.

[5] H. Y. Alkahby, F. N. Jalbout, and M. A. Mahrous, Numerical studies of vertically propagating acoustic and magneto-acoustic waves in an isothermal atmosphere (II), Proceedings of Dynamic Systems and Applications, vol. 3, 2001, pp. 265-272.

[6] H. Y. Alkahby and M. A. Mahrous, On the reflection of Alfvén waves in an ideal magnetoatmosphere, Int. J. Math. Math. Sci. 21 (1998), no. 2, 381-386.

[7] __ Reflection and dissipation of oblique Alfvén waves in an isothermal atmosphere, Int. J. Math. Math. Sci. 22 (1999), no. 1, 161-169.

[8] H. Y. Alkahby, M. A. Mahrous, and A. S. Vatsala, Reflection and dissipation of acoustic waves in an isothermal atmosphere, Proceedings of Dynamic Systems and Applications, vol. 2 (Atlanta, GA, 1995), Dynamic, Georgia, 1996, pp. 45-52.

[9] H. Y. Alkahby and M. Yanowitch, The effect of Newtonian cooling on the reflection of vertically propagating acoustic waves in an isothermal atmosphere, Wave Motion 11 (1989), no. 5, 419-426.

[10] E. R. Priest, Solar Magneto-Hydrodynamics, D. Reidel, Massachusetts, 1984.

[11] M. Yanowitch, Effect of viscosity on gravity waves and the upper boundary condition, J. Fluid Mech. 29 (1967), 209-231.

[12] _ Effect of viscosity on vertical oscillations of an isothermal atmosphere, Canad. J. Phys. 45 (1967), 2003-2008.

[13] _ Vertically propagating hydromagnetic waves in an isothermal atmosphere with a horizontal magnetic field, Wave Motion 1 (1979), no. 2, 123-125.

Hadi Alkahby and ANDRew Talmadge: Department of Mathematics, Dillard UniverSITY, NEW ORLEANS, LA 70122, USA

Abraham Jalbout: Department of Chemistry, University of New Orleans, New ORLEANS, LA 70148, USA 


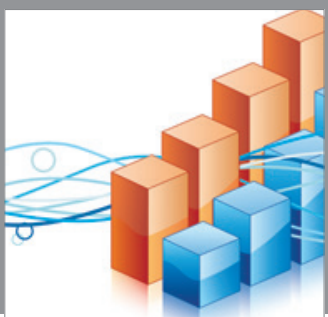

Advances in

Operations Research

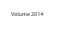

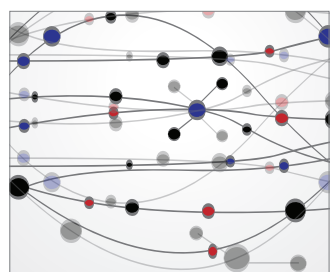

\section{The Scientific} World Journal
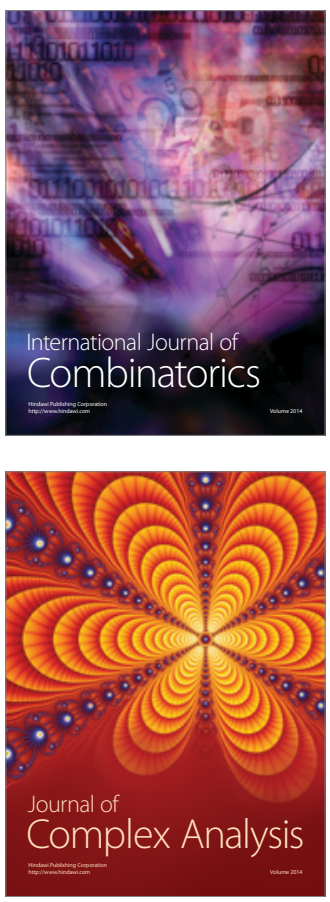

International Journal of

Mathematics and

Mathematical

Sciences
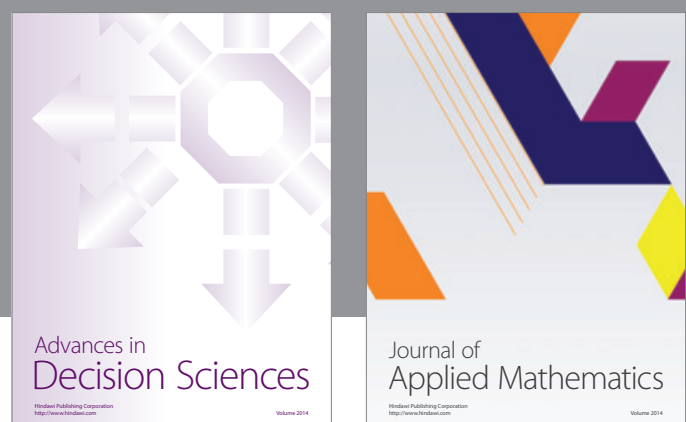

Journal of

Applied Mathematics
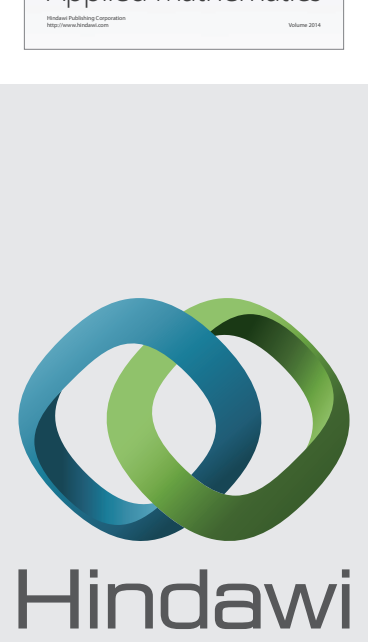

Submit your manuscripts at http://www.hindawi.com
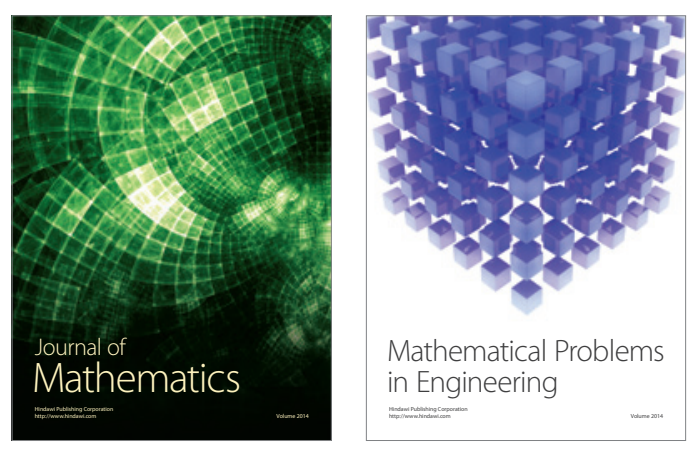

Mathematical Problems in Engineering
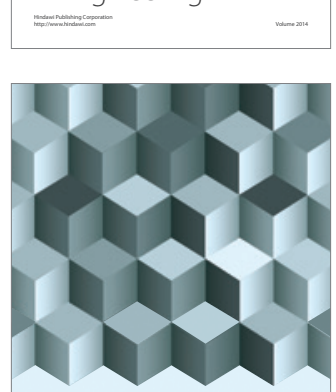

Journal of

Function Spaces
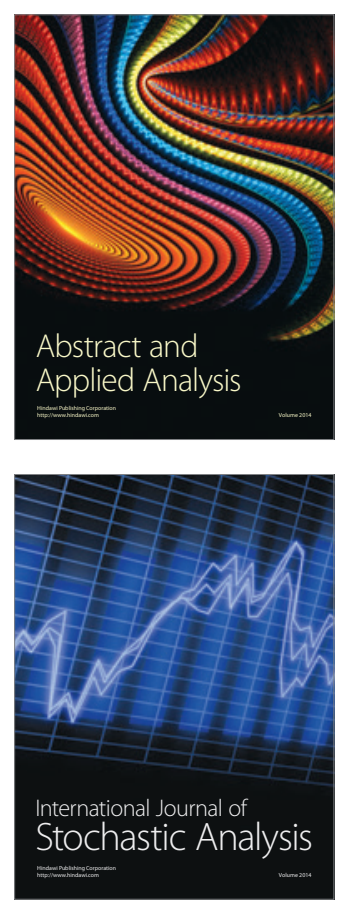

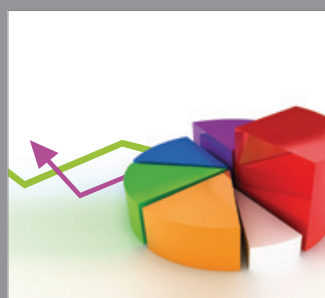

ournal of

Probability and Statistics

Promensencen
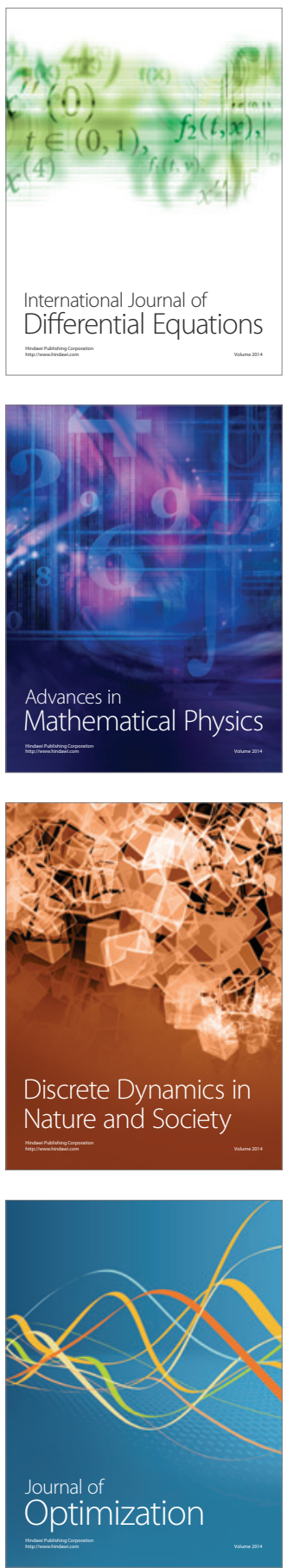